\title{
VIVIENDAS DE BAJO COSTO SISTEMA SALVY
}

\author{
(LOW-COST HOUSING - SALVY SYSTEM) \\ Salvador Suárez Ibarra e Ignacio de Oteiza Sanjosé \\ MARACAIBO - VENEZUELA
}

\section{RESUMEN}

Este trabajo tiene como objetivo presentar una alternativa para la solución del problema actual de la vivienda, a través de un sistema constructivo desarrollado y experimentado en nuestro país -SISTEMA SALVY, dirigido a solucionar el problema de la vivienda de bajo costo, con una tecnología propia que permite la construcción de estas viviendas de una manera rápida, utilizando materiales no tradicionales en construcción pero abundantes en nuestro pais. La vivienda que presentamos permite al usuario ampliarla en altura, de acuerdo a sus necesidades y posibilidades económicas, manteniendo el área libre de la parcela para los denominados huertos del hogar.

\section{SUMMARY}

The aim of this work is to offer an alternative for solving the current problem of housing, through a building system which has been developed and tested in our country - the SALVY SYSTEM.

It is directed towards solving the problem of low-cost housing with own technology, enabling these houses to be built quickly, using materials which are not traditional in building, but are abundant in our country. The house presented by us allows the user to extend its height according to his needs and financial possibilities, keeping the free area of the plot for so-called kitchen gardens.

\section{INTRODUCCION}

El justificante mayor que los entes económicos esgrimen para la no financiación de vivienda de bajo costo, son los riesgos que el capital encara, en las operaciones con grupos sociales de muy bajo poder adquisitivo, y que hacen técnicamente imposible una realización deseada. Son estas organizaciones financieras, las que claman por soluciones tecnológicas más concretas para poder participar decididamente, en la solución de los males, que en esta materia aquejan a los pueblos en desarrollo. Soluciones concretas, en las que el problema técnico vaya acompañado del social y económico en esfuerzo unisono, donde estos factores concuerden en acertadas realidades.

El sistema que presentamos es el mismo que en Venezuela trata de erradicar definitivamente, la presencia del rancho. Ha sido estudiado teniendo en cuenta el medio social al cual va dirigido, con sus complejos problemas y realidades. Por esta razón hemos adoptado técnicas de fácil entendimiento, al alcance de cualquier capacidad y que conlleven calidad y economia. Técnicas confiables para que lleguen a financiarse por esos organismos que requirieron de ellas. Estos sistemas se caracterizan por la industrialización parcial, que comprende la formación estructural de la vivienda y una aplicación directa en obra, de ciertos materiales componentes de la misma, con procedimientos ágiles y eficaces.

Hemos procurado en nuestros sistemas constructivos, que la participación del hombre sea decisi- va, midiéndolo por producto y calidad, y valorando asi su capacidad inventiva y no la energia que origina. Con nuestros métodos constructivos adquirirá conocimientos que lo presentan como un ser habilidoso. En materia de construcción no siempre es la máquina la que acelera y economi$z a$, siendo esto confirmado en la disposición de nuestros sistemas, donde el hombre interviene, y con materiales y métodos apropiados compite y descalifica a la máquina, consiguiendo costos de producción más en consonancia con los requerimientos de la vivienda del marginado.

Las problemáticas grúas y su más problemático manejo y mantenimiento, han sido eliminadas, al hacer uso de materiales para edificar, livianos y manejables.

En la construcción tradicional estos grupos marginados obtenian periódicamente sustento y ayuda por algún tiempo. Son grupos difíciles de contratar por su bajo nivel cultural, siendo por tanto sus actividades muy reducidas e inestables.

Con los sistemas que presentamos este ser humano se integra a nuestra sociedad, con conocimientos y logros en nuevas artes que están produciendo cambios innovadores y radicales de las formas como se piensa y actúa en materia constructiva.

En Venezuela hemos aplicado y aplicamos para amortización de estas populares viviendas métodos de plantaciones horticolas por medio de barbacoas, que situadas en los patios de las mismas 


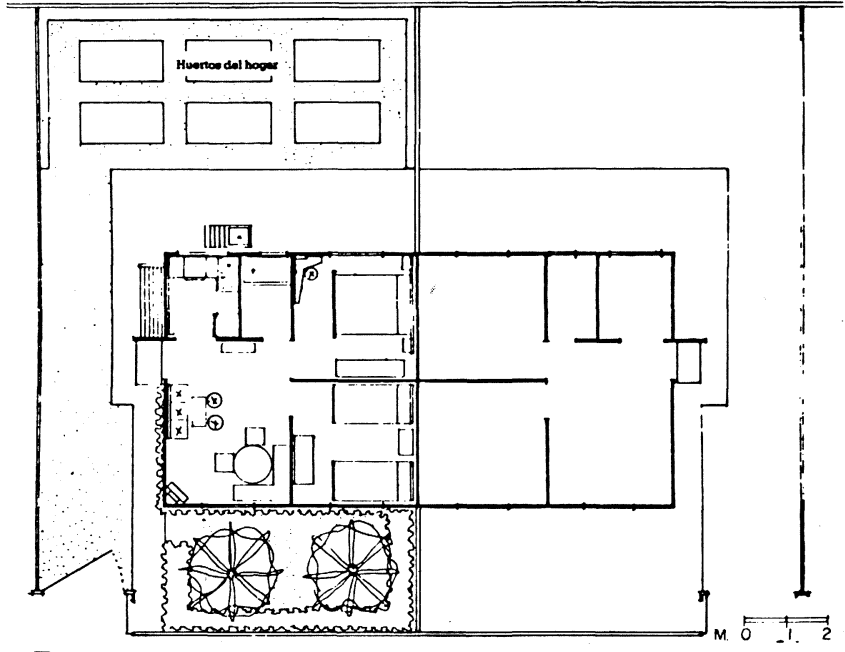

Esquema 1.

son cultivados por sus moradores, obteniendo beneficios apropiados para hacer frente a obligaciones contraídas en la adquisición de la vivienda.

La participación del usuario en la construcción de esta morada, genera medios económicos para enfrentarse con gastos iniciales. Estas actividades entran también en la aportación de técnicas y procedimientos para que el hombre marginado sea poseedor de una vivienda, de acuerdo a su dignidad humana. (Esquema 1).

\section{EL PROBLEMA DE LA VIVIENDA EN VENEZUELA}

En nuestro pais el problema de la vivienda se hace cada vez más crítico, en los últimos doce años (1971/1983), el déficit global ha aumentado en 265.000 viviendas, para llegar a un total de 1.100 .000 actualmente, lo que significa que un $37 \%$ de las familias en Venezuela están sin vivienda, cifra alarmante para un país que ha contado en las últimas décadas con recursos económicos privilegiados y con una masiva inversión de fondos públicos al sector de vivienda, tanto al Instituto Nacional de la Vivienda (INAVI), como para la estimulación a empresas privadas a través de subsidios a la producción y financiamiento de vivienda para la clase media.

El Instituto Nacional de la Vivienda es el organismo encargado de ejecutar el programa del gobierno nacional, en sus 55 años de funcionamiento se pueden distinguir tres momentos de acción en materia habitacional. Una primera etapa 1928/1950 con una acción limitada por carencia de recursos y orientada a la construcción de viviendas unifamiliares aisladas, en las mayores ciudades del pais.

Una segunda etapa 1950/1958, marcada por la dictadura de Pérez Jiménez con suficientes recursos económicos y enfocando la construcción de vi- viendas hacia superbloques, ocasionando graves problemas sociales y trastornos de conducta en las comunidades.

La tercera etapa 1958/1983, tras una paralización al principio de ésta debido a la recesión económica que vivía el pais, observamos diversos planes con recursos abundantes, acelerando la construcción de viviendas, haciendo participar decididamente a la empresa privada. Es en el periodo $1970 / 1974$ cuando se manifiesta, que los sistemas constructivos tradicionales son ineficientes frente a las metas establecidas de construcción masiva, promoviendo métodos industrializados y de prefabricación que hasta ese momento no habian sido atendidos debidamente, a pesar que en nuestro país es alrededor del año 1965 cuando comienza la era de la prefabricación y con ella una descomunal carrera hacia la adquisición de tecnologia foránea, que tantos males causó y sigue causando. En un afán de competir en estos millonarios planes de vivienda, las empresas constructoras recurrieron a los más variados procedimientos para edificar, sin pensar debidamente la eficacia de los mismos en un medio como el nuestro. Sistemas foráneos ya caducos en sus paises de origen, se introducen en Venezuela invirtiendo grandes capitales para la construcción de estas industrias e importación de equipos.

A pesar de estos intentos nos damos cuenta que el problema habitacional lejos de mejoras ha ido agudizándose, en estos momentos de grave crisis económica en nuestro país, nos encontramos ante un gran reto, solucionar el déficit de vivienda en el menor tiempo posible. Estamos convencidos que este reto que se nos plantea, puede llevarse a cabo con el incentivo del desarrollo de tecnologias apropiadas, que busquen: una velocidad de producción, una reducción de costos y que abra posibilidades al desarrollo y puesta en el mercado de nuevos materiales y técnicas acordes con la estructura de la industria. Estas soluciones estarán adaptadas a las necesidades prioritarias de nuestra sociedad, a la mano de obra con que contamos, industrializando la construcción de componentes de la vivienda, sin que estas industrias sean sofisticadas, ni necesiten grandes inversiones de capital. Planteando también acciones concretas por parte del gobierno y de los entes financieros de acuerdo a las posibilidades reales de la población mayoritaria en Venezuela.

\section{CARACTERISTICAS ESPACIALES DE LA VIVIENDA (Esquema 2)}

- Area cubierta: $49 \mathrm{~m}^{2}$

- Area interna: $36 \mathrm{~m}^{2}$ 


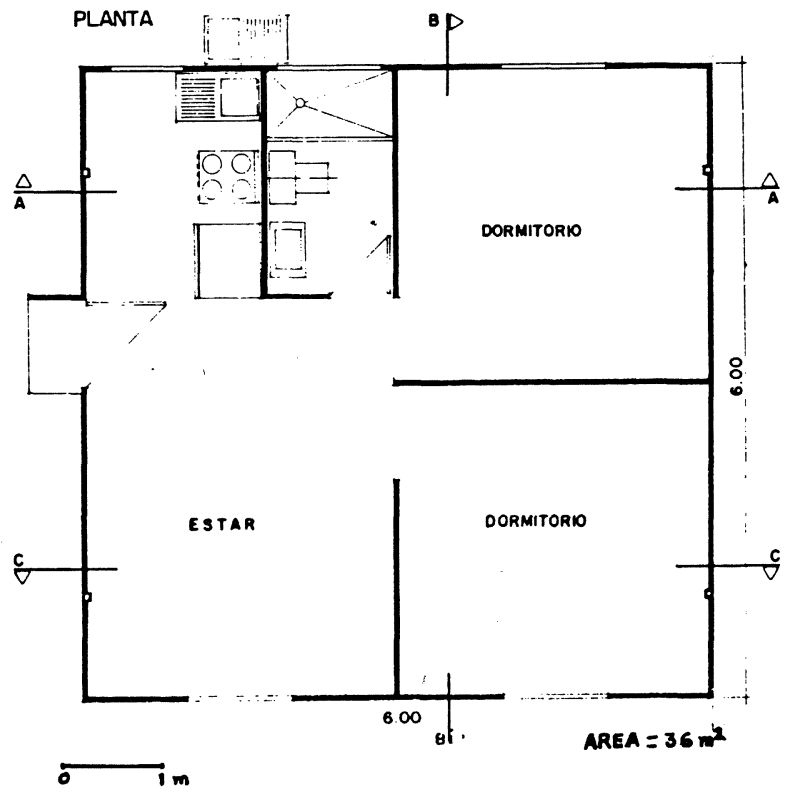

FACHADA LATERAL

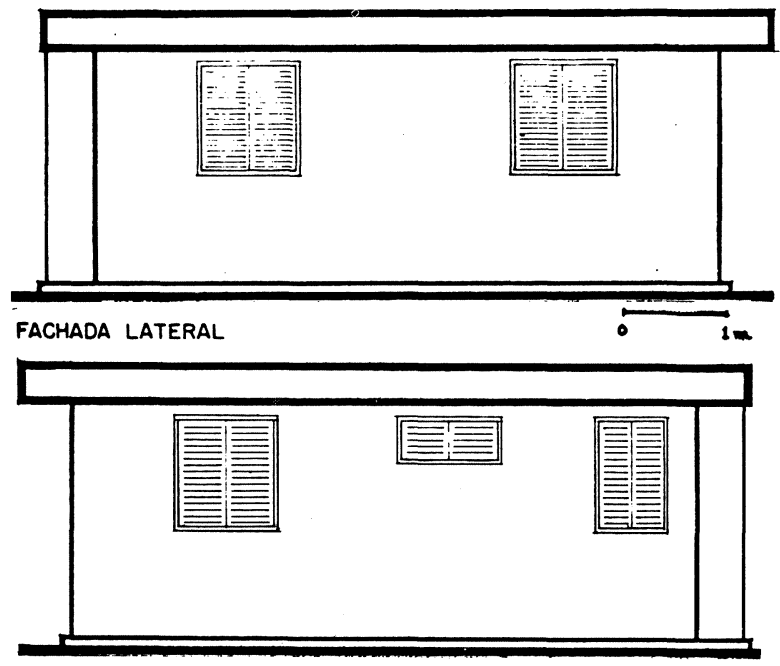

Esquema 2.

- Espacios de la vivienda:

Estar

2 Dormitorios

Sala sanitaria

Cocina

Lavadero exterior

\section{DESCRIPCION DEL SISTEMA}

El sistema que presentamos se basa fundamentalmente en dos componentes que describimos a continuación, que son la estructura y los cerramientos, los cuales trabajando solidariamente conforman una construcción de características sobresaliente en cuanto a:

- Resistencia Mecánica, permitiendo crecimiento en altura.
- Construcción liviana.

- Comportamiento antisísmico.

- Construcción antitérmico - antiacústica e impermeable.

- Fácil montaje sin necesidad de equipar mano de obra especializada.

- Participación del usuario en el diseño de los espacios, adecúandose los mismos a las actividades y necesidades en el tiempo de las personas que habitan en la vivienda.

- Bajos costos.

\section{a) LA ESTRUCTURA}

La estructura de la vivienda está conformada por una MALLA ESPACIAL de $7 \mathrm{~m} \times 7 \mathrm{~m}, 4$ Columnas tubulares de 3 " $x$ 3" y cimentaciones aisladas 0 pilotes.

La Malla Espacial es el elemento fundamental de la estructura, tiene la característica de estar formada por dos retículas tubulares unidas entre si por diagonales. Las retículas se componen de un conjunto de cuerdas paralelas y distanciadas un metro entre si, y por conjunto perpendicular a las anteriores y con las mismas características geométricas. Estas cuerdas nos permiten colgar tabiques o cerramientos en las más variadas posiciones.

La Malla Espacial se arma sobre la losa de piso, totalmente nivelada y con cuatro columnas ancladas en sus respectivas bases. Una vez armada la malla se procede a su elevación hasta la altura prevista, utilizando para esto aparejos sencillos colocados en las columnas. Ya elevada y sujeta por medio de soldadura en su altura correspondiente, se comienzan a colocar los tabiques y la cubierta propiamente dicha. Característica resaltante de esta malla espacial es su poco peso, que incide favorablemente en las dimensiones de las bases y de las columnas, así como su resistencia mecánica, ya que la misma está en capacidad de cumplir la función de entrepiso para la etapa de ampliación de la vivienda. (Esquema 3).

\section{b) LOS CERRAMIENTOS}

Los cerramientos en nuestra solución, se basan en la idea fundamental de colgar tabiques (membrana) y no levantar tabiques como en los sistemas tradicionales o en los sistemas prefabricados.

Teniendo la reticula de la malla espacial contamos con un elemento suficientemente resistente, donde se cuelgan los tabiques o cerramientos. 
(B)

MALLA DE CUBIERTA

(4)
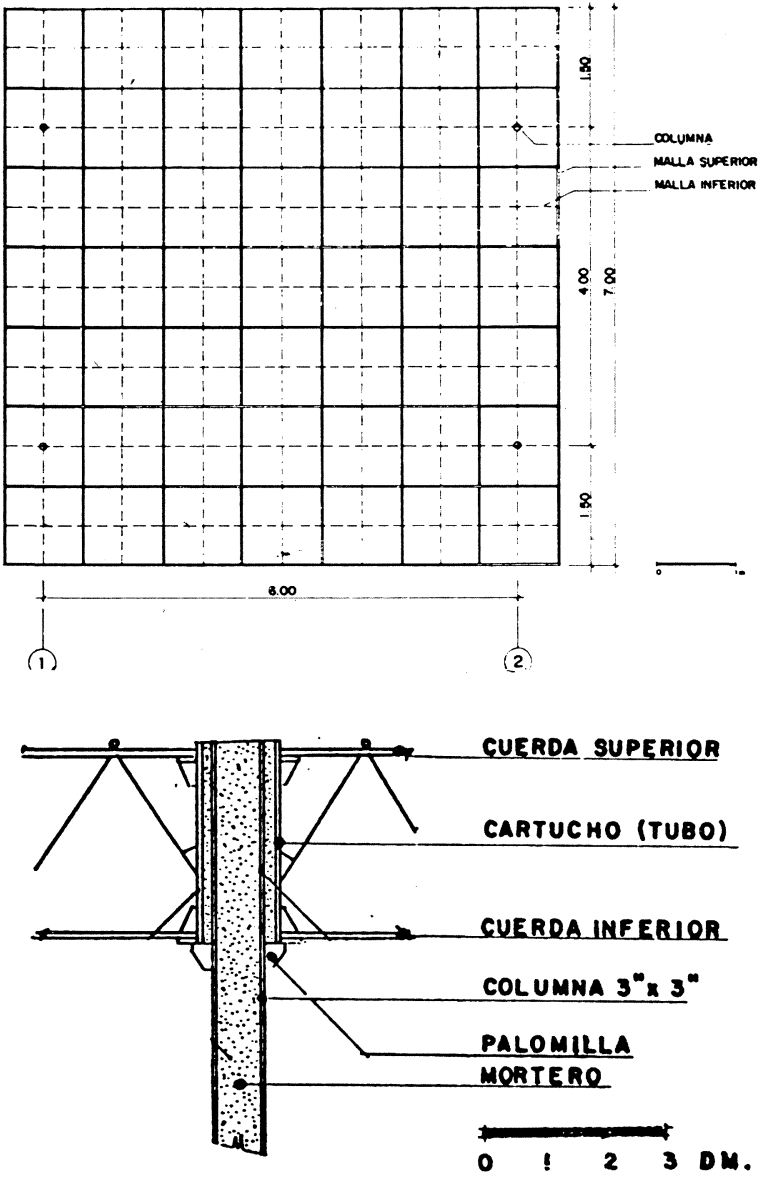

EMPOTRAMIENTO EN LA CUBIERTA

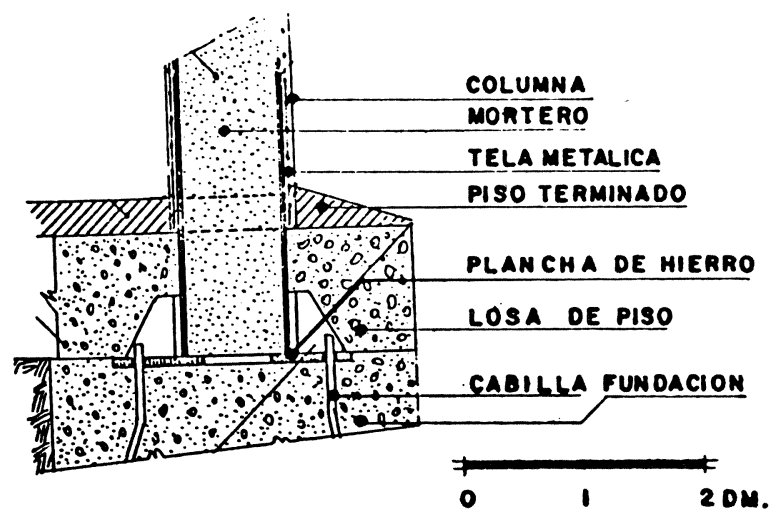

ANCLAJE DE COLUMNA

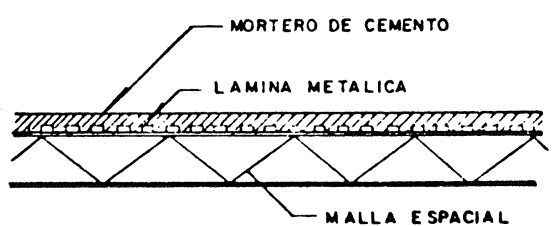

DETALLE DE LA CUBIERTA

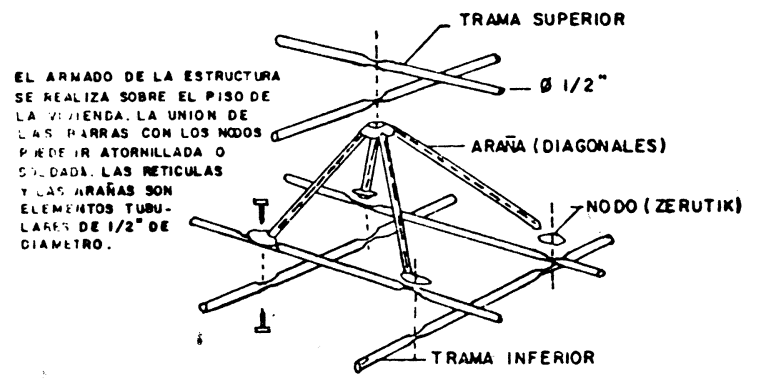

DESPIECE DE LOS ELEMENTOS DE LA MALLA ESPACIAL SISTEMA SALVI TPO ZERUTIK
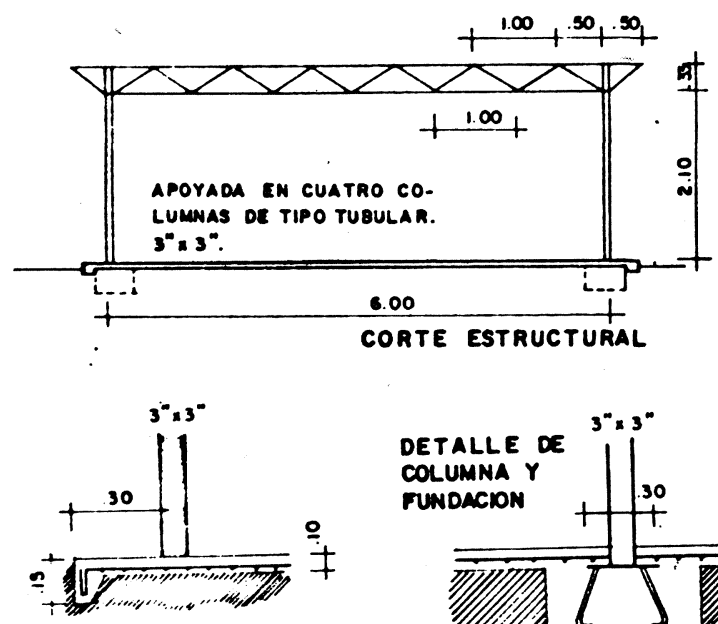

DETAUE DE PLPO

PL'PISO TIENE UN ESPE.

SOR DE $.10 \mathrm{cmo}$. Y ESTA REMATADO CON UN BAOCAL A FIN DE EVITAR LA EROSION EN LA BASE.

LAS COLUMAMS ESTAN UNIDAS A UNA PLAMOHA METALICA Y ESTA A SU

VEZ AL EMPARAULLAOO oE la Fundacion.
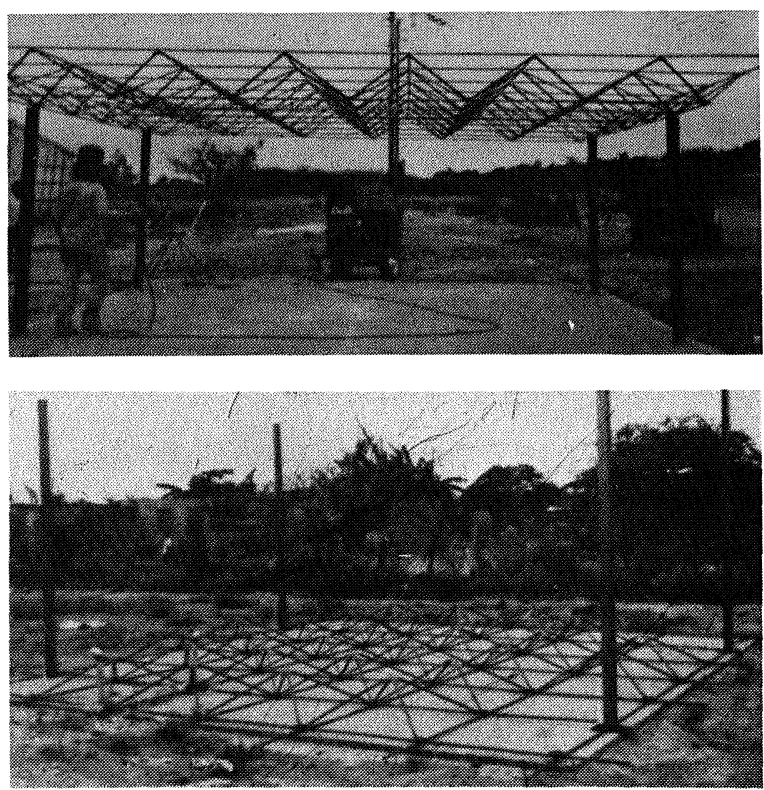

Esquema 3. 
Los cerramientos del sistema son de tipo "SANDWICH" compuestos por un alma o regrueso que puede ser de diversos materiales de acuerdo al medio como: Poliestireno, poliuretano, fibras o lanas minerales, desechos industriales, desechos agricolas, arcillas expandidas, etc.; en nuestro medio utilizamos preferentemente el poliestireno en láminas, por ser un material derivado de nuestra industria petroquimica, con excelentes caracteristicas antitérmicas y antiacústicas, a la que liviano y fácil de instalar. Este alma o regrueso se encuentra entre bastidores y telas metálicas en ambas caras, cosidas entre si y tensadas entre la malla, los bastidores y el piso.

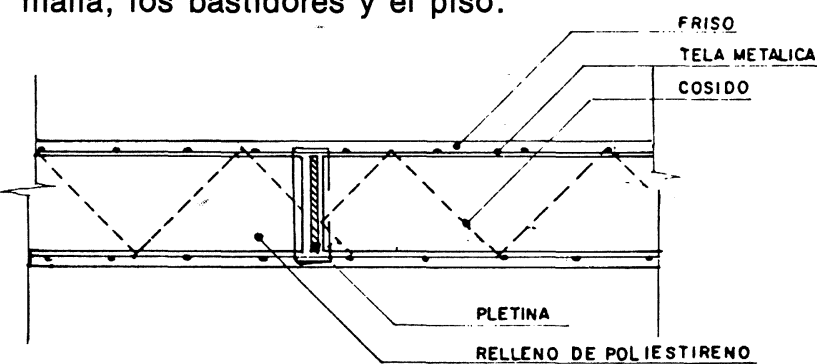

CONEXION TABIQUES CONTINUOS

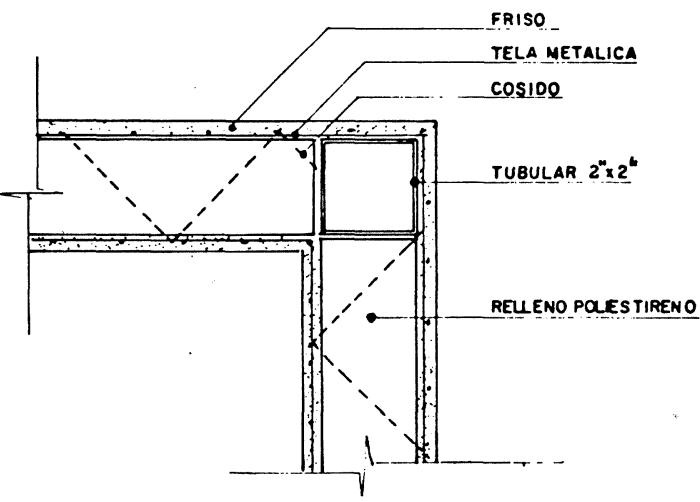

CONEXION TABIQUES EN ANGULO
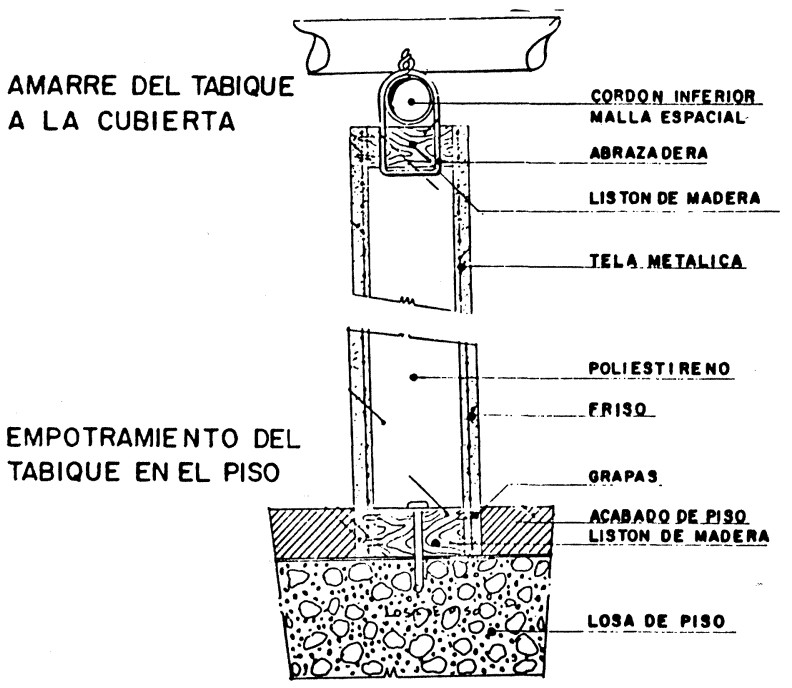

Esquema 4.

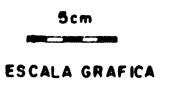

Sobre estas telas metálicas se aplica un friso por procedimientos tradicionales o proyectando mecánicamente morteros a base de cemento y arena. Estos cerramientos han sido analizados con pruebas de impacto, dando resultados muy superiores a los realizados con elementos alfareros.

Los cerramientos internos pueden ser modificados, adaptándose al espacio que el usuario requiera en etapas posteriores para ampliar la vivienda. Con la malla espacial como cubierta o entrepiso, y afianzados los cerramientos a esta estructura, al piso y a los bastidores se constituye una construcción monobloque, con características antisismicas y de alta resistencia a embates climatologicos. (Esquema 4).

\section{c) CIMENTACIONES Y LOSA DE PISO}

Cimentaciones aisladas para recibir las cuatro columnas metálicas de 3" x 3" macizadas con mortero.

La losa de piso será de hormigón armado de $0,10 \mathrm{~m}$ de espesor.

\section{d) LA CUBIERTA}

La cubierta propiamente dicha, se realiza con láminas metálicas corrugadas apoyadas sobre las cuerdas de la malla espacial. Utilizando las láminas como encofrado perdido, se vacia sobre las mismas un mortero de cemento de $5 \mathrm{~cm}$ de espesor, el cual está armado con telas metálicas. Dispuesta la cubierta de esta forma cumplirá la función de entrepiso para la etapa de crecimiento en altura de la vivienda. (Esquema 5).

\section{e) PUERTAS Y VENTANAS}

Las puertas y ventanas podrán ser metálicas 0 de madera, y los marcos en cada uno de los casos irán sujetos a bastidores metálicos embutidos en los cerramientos. En gráficos adjuntos describimos soluciones de marcos y su integración al tabique. (Esquema 6).

\section{f) INSTALACIONES}

Las instalaciones sanitarias se realizarán siguiendo las normas establecidas por el arte. Se construirá un tabique sanitario con las mismas características del anteriormente descrito. Las piezas sanitarias y las tuberías aparecen en el gráfico inferior.

La electricidad se realizará superficialmente, con un punto de luz y tomacorrientes en cada uno de los espacios de la vivienda. (Esquemas 7, 8 y 9). 
VIVIENDA UNICA

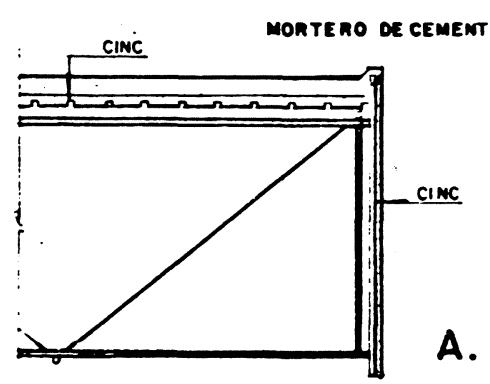

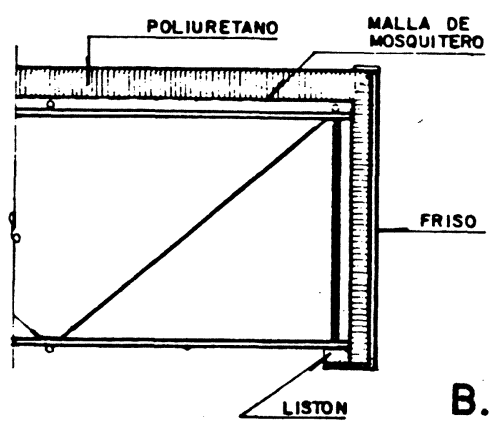

B.
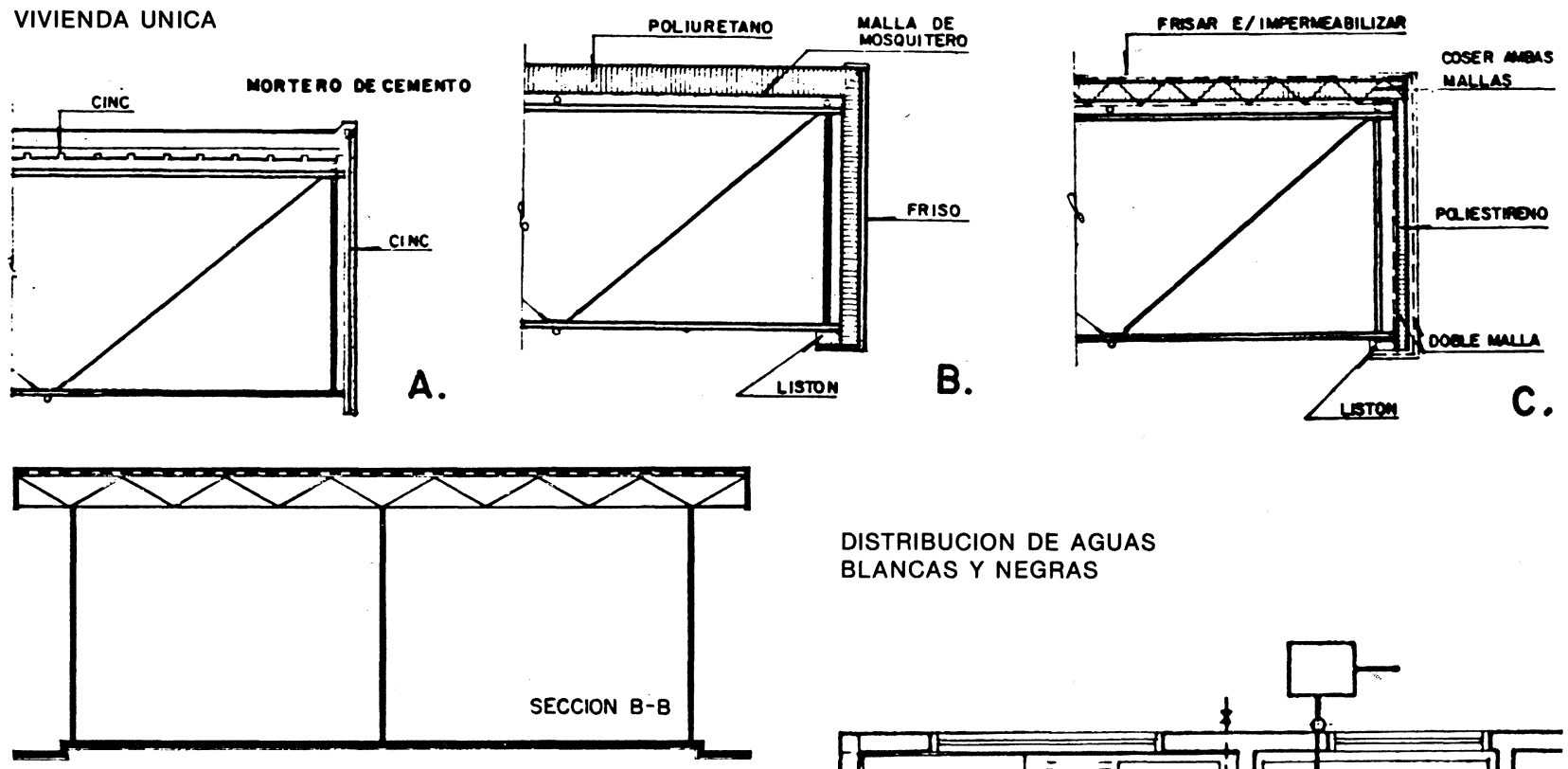

Esquema 5.

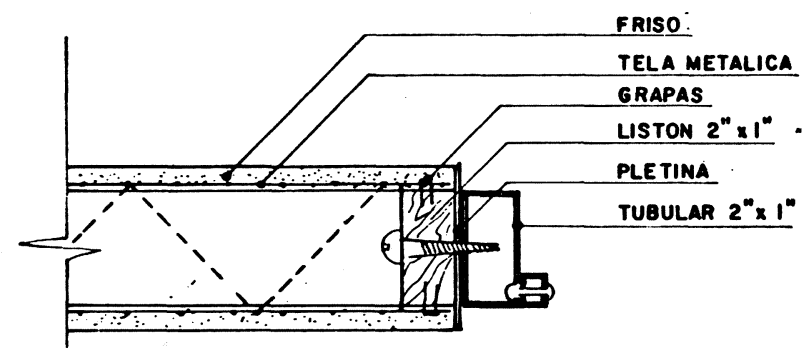

MARCO DE PUERTA (metálico)

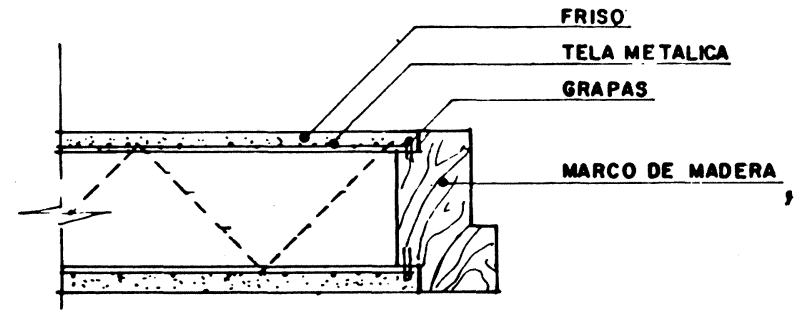

MARCO DE PUERTA (madera)

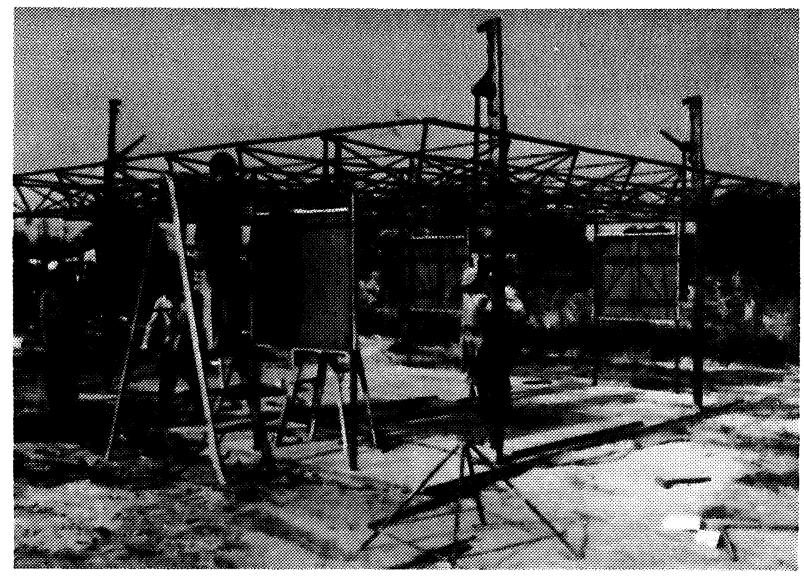

Esquema 6.

DISTRIBUCION DE AGUAS

BLANCAS Y NEGRAS
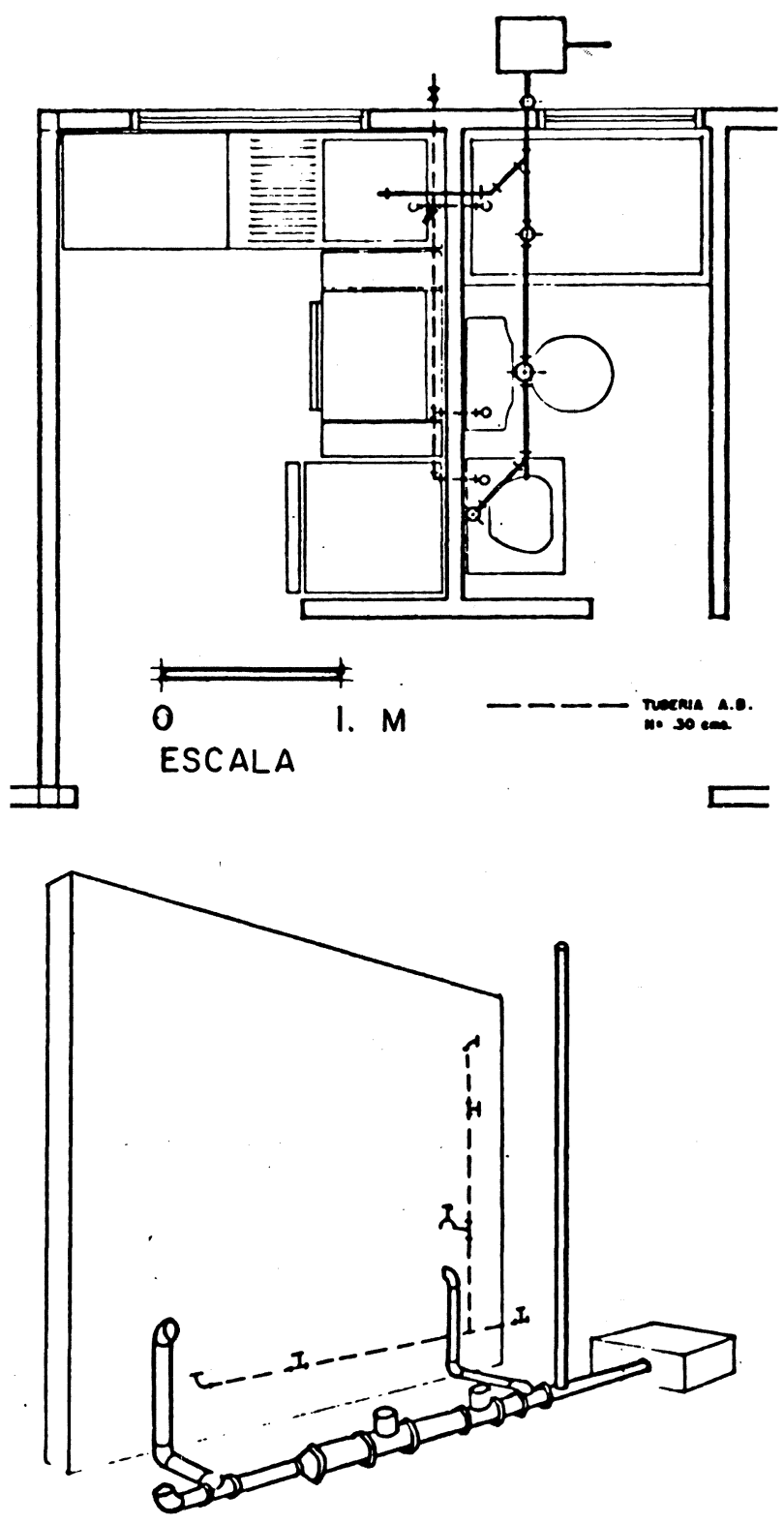

Esquema 7. 


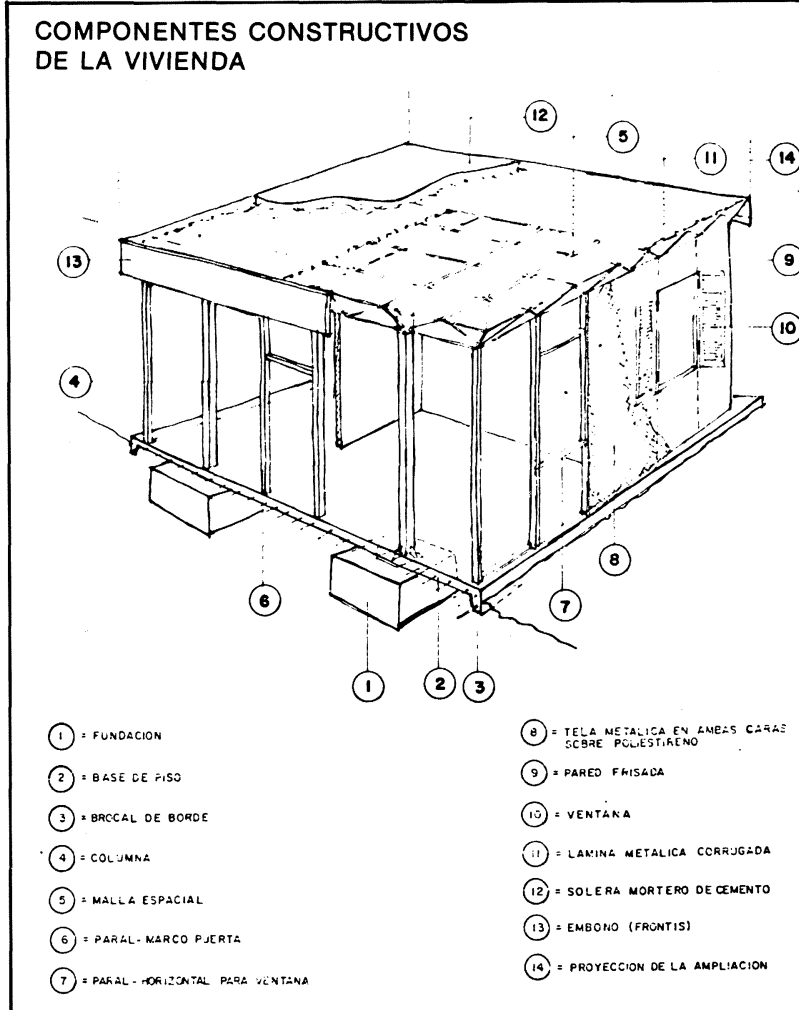

Esquema 8.

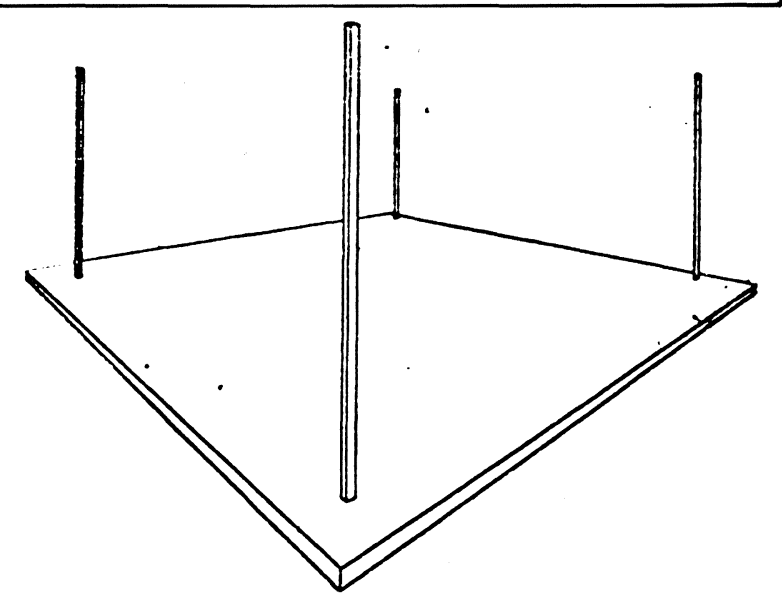

VACIADO DE LOSA DE PISO Y ANCLAJE DE COLUMNAS

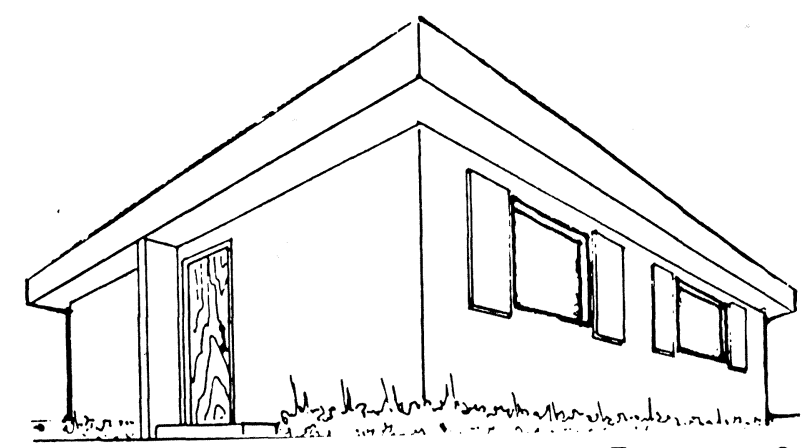

5. VIVIENDA UNICA

Esquema 9.

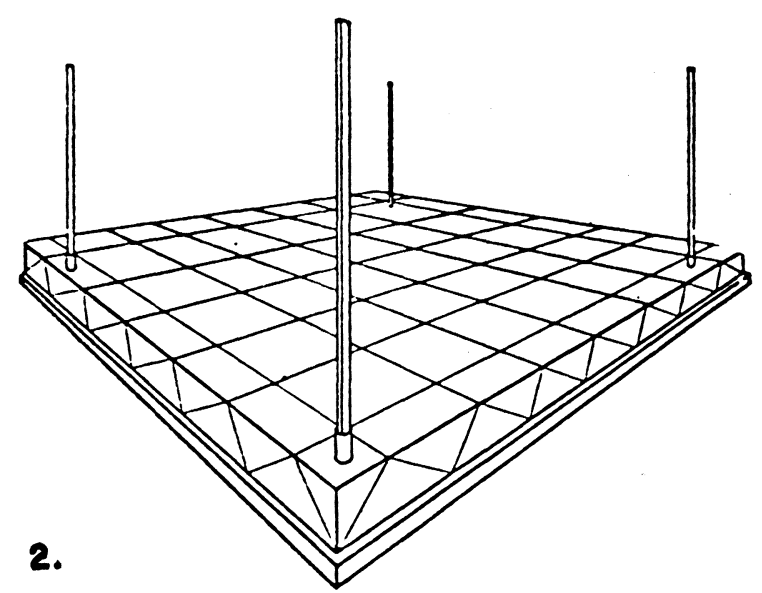

MALLA ESPACIAL DISPUESTA A SER ELEVADA

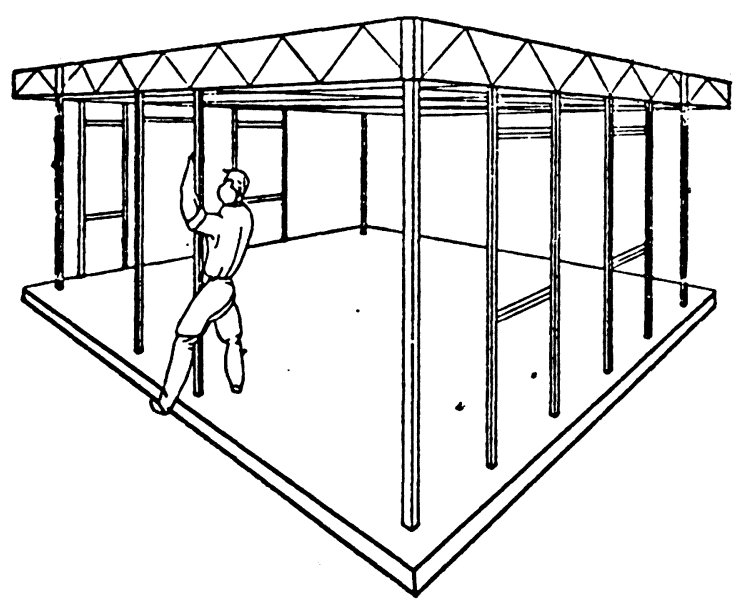

3.

MALLA ESPACIAL EN SU ALTURA

Y COLOCACION DE MARCOS

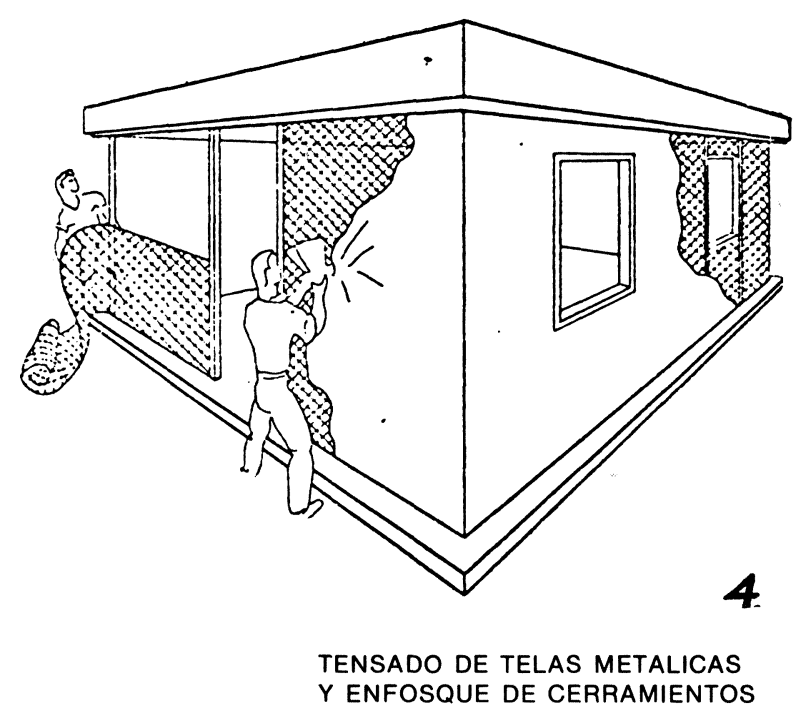




\section{AMPLIACION DE LA VIVIENDA}

Rompiendo con el esquema tradicional en el cual se realiza la vivienda como un producto terminado, nos decidimos a ofrecer en nuestra propuesta para la vivienda de bajo costos, una solución que permita e incentive el MEJORAMIENTO Y LA AMPLIACION progresiva de acuerdo a la capacidad económica del usuario y mediante un proceso de AUTO-CONSTRUCCION.

Esta vivienda UNICA en un principio por su estandarización, se convertirá en UNICA otra vez, dadas las posibilidades y variantes que ofrecen estas viviendas en el orden arquitectónico.

Construidas las viviendas únicas como solución emergente a un problema social que abate tanto a nuestro país como a todos los países en desarrollo, se han dispuesto en ellas particularidades constructivas con resultados prácticos y positivos ya experimentados.

La monotonia de formas y colores de la vivienda podría influir en el orden social ya que el individuo no se identificaría con ella, esto no sucederá por las posibilidades futuras de ampliación que permite nuestra solución constructiva.

La malla espacial es la que hace posible las ampliaciones sobre ella de acuerdo a las necesidades propias de la evolución social del usuario, sin menoscabo de su resistencia mecánica y permitiendo el uso racional de la parcela.

De aqui saldrá una arquitectura razonada y funcional nacida de un pueblo y que pudo superar una etapa crítica.

Ofrecemos a continuación algunas variantes que muestran estas viviendas en el orden arquitectónico, conscientes de las infinitas soluciones que el pueblo ha demostrado tener. (Esquema 10).

EJEMPLOS DE AMPLIACION DE LA VIVIENDA UNICA

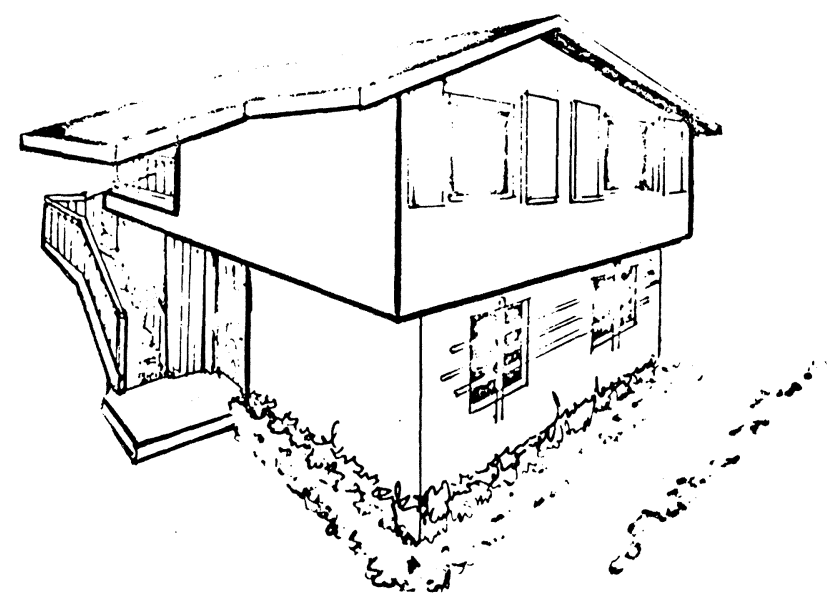

\section{ESTIMACIONES DE COSTOS}

Son diversos los organismos internacionales que se encargan de investigar el problema de la vivienda de bajo costo. UNCH-Habitat organismo dispuesto por las Naciones Unidas, plantea en lo referente al tipo de vivienda a estudiar, aquella que esté entre los 30 y $50 \mathrm{~m}^{2}$ y cuyo costo sea inferior a los CIEN DOLARES USA por metro cuadrado. (USA $\left.\$ .100 / \mathrm{m}^{2}\right)$.

El costo de estas viviendas es estimado en QUINIENTOS BOLIVARES $/ \mathrm{m}^{2}$. (Bs. $500 / \mathrm{m}^{2}$ ), y el área de la denominada por nosotros vivienda única es de $36 \mathrm{~m}^{2}$. En Venezuela construimos viviendas de este tipo, terminadas con un costo total de DIECIOCHO MIL BOLIVARES (Bs.18.000,00) que equivale a MIL OCHOCIENTOS \$.USA. (\$USA.1,800). (Ver fotos página siguiente).

\section{BIBLIOGRAFIA}

- SUAREZ I, S. y DE OTEIZA S, I. 1980. SISTEMA SALVY. Búsqueda de la Vivienda Popular. Ponencia Simposium "La Vivienda, problemas y soluciones". Maracaibo - Venezuela.

- SUAREZ I, S. 1983. Estudio de Producción en viviendas para grupos marginales. Diario «El Zulianon. 17 Abril. Maracaibo. Venezuela.

- SUAREZ I, S. 1984. Tecnología Nuestra. Monografia. Maracaibo - Venezuela.

- SUAREZ I, S. 1984. Plan nacional de viviendas de bajo costo. Maracaibo - Venezuela.

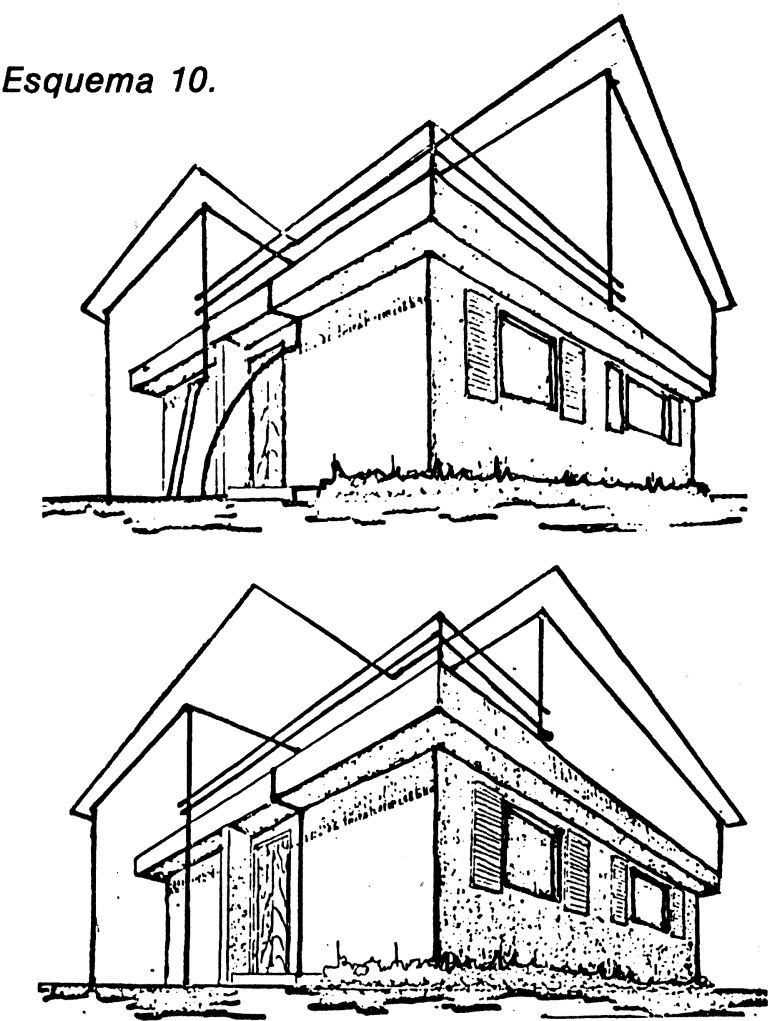



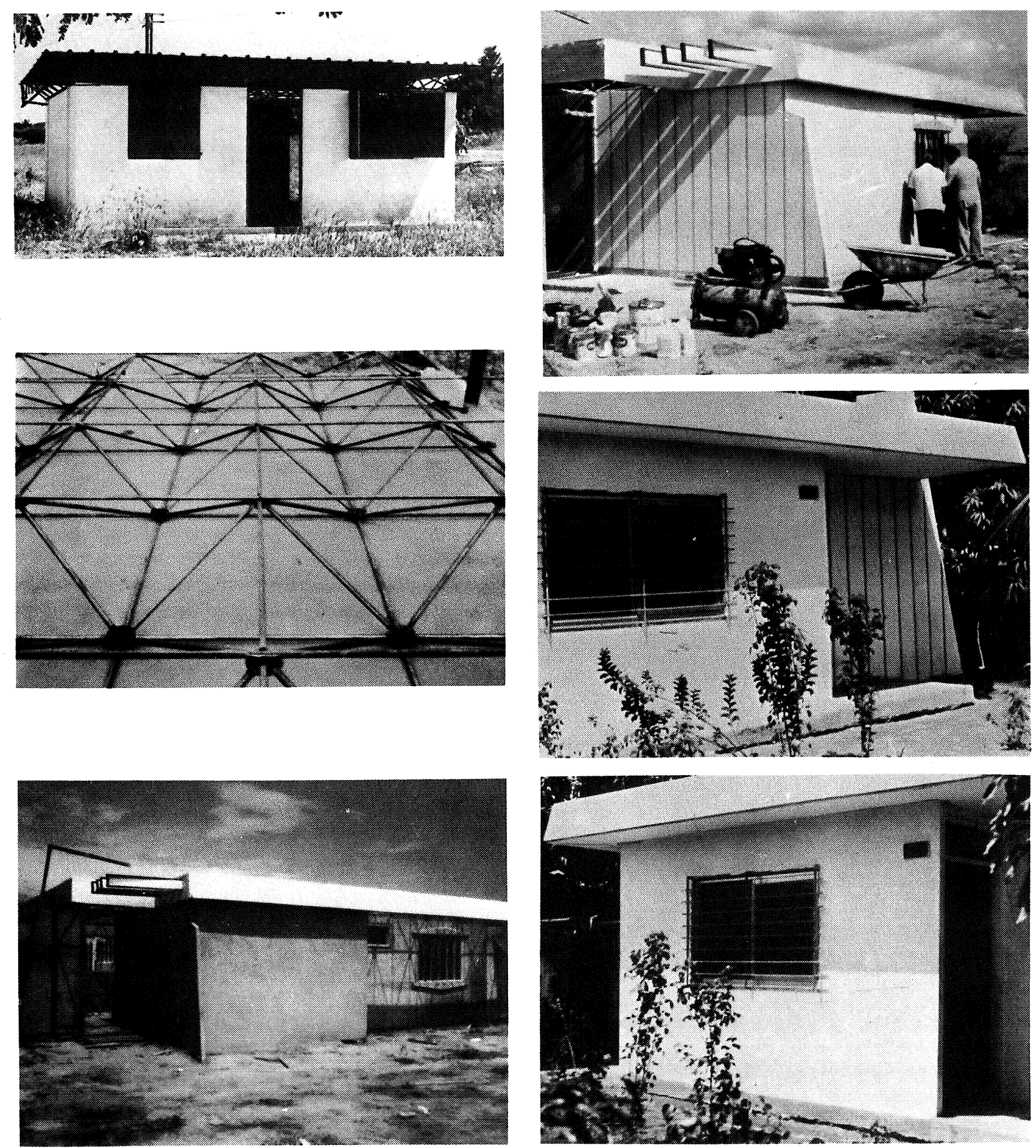

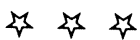

